\title{
ROBERT FRANK
}

\section{DER ÉLYSÉE-VERTRAG: EIN DEUTSCH-FRANZÖSISCHER ERINNERUNGSORT?}

Nachdem in den vorangegangenen Beiträgen die verschiedenen Aspekte des Élysée-Vertrages analysiert worden sind, stellt sich nun die Frage, welchen Platz der Vertrag in der Erinnerung der Franzosen, der Deutschen und der Europäer einnimmt. Bis 1963 hatten Frankreich und Deutschland eine gemeinsame Geschichte, die jedoch nicht notwendigerweise zu einer gemeinsamen Erinnerung führte. In der Folge der verschiedenen Kriege besaßen die beiden Länder eine gemeinsame Geschichte, die sich zwischen sie gestellt und belastet hatte. Siege auf der einen, Niederlagen auf der anderen Seite erschwerten es Franzosen und Deutschen, eine gemeinsame Erinnerung zu besitzen. Es ist schwierig, eine geteilte Erinnerung von wechselseitigen Blutbädern zu haben. Die kollektive Erinnerung ist an eine kollektive Identität gebunden, die auf dem Gefühl beruht, einer Gemeinschaft anzugehören. Nun beruht dieses $\mathrm{Zu}$ sammengehörigkeitsgefühl aber auf Gefühlen, Liebe, Tod, Blut und Tränen; und die Gemeinschaft, mit der sich das Individuum am stärksten identifiziert, ist die Nation, sieht man einmal von privaten Gemeinschaften wie der Familie ab. So bleibt die Nation die bedeutendste öffentliche Erinnerung, und es scheint, daß für eine binationale Erinnerung wenig Platz zur Verfügung steht, weil sie nicht in der Lage ist, geteilte Gefühle und eine gemeinsame Erinnerung hervorzubringen. Dies gilt um so mehr für Nationen, die sich regelmäßig in Kriegen gegenüberstanden.

Sicherlich folgt auf Krieg Frieden, und Frankreich und Deutschland weisen eine gemeinsame Friedensgeschichte auf. Doch war es schwierig, darauf eine gemeinsame Erinnerung aufzubauen, denn nach 1871 blieb der Frieden für Frankreich schmerzhaft, während er Deutschland den Sieg und die Einheit brachte, und nach 1918/19 wollte Deutschland den Frieden nicht anerkennen, nicht zuletzt weil er Frankreich begünstigte. Der Frieden war eben nur das Ende der Kriegshandlungen bzw. der Beginn einer Zwischenkriegsphase; das Bemühen um Annäherung und Versöhnung gab es bis zur Ära Briand-Stresemann (1925-1929) nicht. Diese wenigen Jahre reichten jedoch nicht aus, um eine tiefe und dauerhafte Erinnerung in den nationalen Erinnerungslandschaften zu verankern und eine binationale Erinnerung an den Frieden aufzubauen.

Die Situation hat sich mittlerweile zweifellos geändert, seit der Frieden zwischen beiden Ländern nicht mehr nur ein Waffenstillstand zwischen zwei 
Kriegen darstellt, seit er auf beiden Seiten des Rheins mit dem Willen verbunden ist, eine gemeinsame Zukunft zu gestalten, einen Rahmen für eine Schicksalsgemeinschaft und einen Grundstein für die Europäische Integration zu schaffen. Für diese Veränderungen war der Vertrag von 1963 sicherlich unentbehrlich. War er aber auch ein »Erinnerungsort" im Sinne von Pierre Nora, ein Ort, in dem sich die "Erinnerung kristallisiert und Zuflucht sucht «? ${ }^{1}$ Um auf diese Frage eine Antwort zu geben, wollen wir einen Blick auf die Definition werfen, die der Urheber dieser Formel vorschlägt:

Ein Erinnerungsort ist eine materielle oder immaterielle Bedeutungseinheit, die durch menschlichen Willen oder mit der Zeit zu einem symbolischen Element des Erinnerungserbes einer Gemeinschaft [in diesem Fall der französischen Nation; R.F.] geworden ist. ${ }^{2}$

Ist der Vertrag von 1963 »zu einem symbolischen Element des Erinnerungserbes" einer deutsch-französischen Gemeinschaft geworden? Hat die Zeit die Wunden geheilt, um eine solche Gemeinschaft zu schaffen und aus dem Vertrag eines ihrer Symbole zu machen? Gibt es den Willen, um aus ihm einen Erinnerungsort werden zu lassen? Hat sich also ein binationaler Wirkungsprozeß um diesen Vertrag herum in Gang gesetzt, der sowohl 1950 als auch 1963 noch unmöglich erschien?

Infolge der Wirkungskraft und der herausragenden Stellung der nationalen Erinnerungslandschaften gab es in den 20 Jahren nach der Vertragsunterzeichnung keinen Platz für eine binationale Erinnerung oder einen binationalen Erinnerungsort. Es bedurfte erst der Zeit und dem politischen Willen der Regierungen, damit sich die Situation in den 1980er Jahren änderte. Wirkungsvolle Symbole und manche Mythen wurden geschaffen, um den symbolischen Gebrauch der deutsch-französischen Vergangenheit zu modifizieren.

\section{Die Wirkungsmacht der Zeit: von der Gewohnheit bis zur Erinnerung}

Kann ein Datum (22. Januar 1963) ein Erinnerungsort werden? Dazu bedarf es bestimmter Bedingungen: die Wirkungsmacht der Zeit und einer plausiblen Übereinstimmung zwischen der Vergangenheit, dem Erinnerungsobjekt und der Gegenwart, die diese Erinnerung aktiviert oder reaktiviert. In diesem Fall ermöglicht die verstreichende Zeit, daß ein Wille zur Erinnerung einsetzen kann. Der 14. Juli ist ein gutes Beispiel für solch einen Prozeß: Er wurde erst im Jahre $1880 \mathrm{zu}$ einem wirklichen Erinnerungsort, als die wiedergeborene

Pierre Nora (Hg.), Les lieux de mémoire, Bd. 1: La République, Paris 1984, S. XVI.

2 DERS., From lieux de mémoire to realms of memory, in: DERS., Lawrence KRITZMAN (Hg.), Realms of Memory: Rethinking the French Past, Bd. 1: Conflicts and divisions, New York, Chichester 1996, S. XVII. 
Republik sich gefestigt hatte und diesen Tag $\mathrm{zu}$ ihrem Nationalfeiertag machte.

Von Beginn an stellten sich die Grundbedingungen für die Erinnerung an den Élysée-Vertrag jedoch schwierig dar. Die oben beschriebene Beziehung zwischen feierlich begangener Vergangenheit und feierlich zu begehender Gegenwart gestaltete sich besonders in den ersten Jahren nach der Vertragsunterzeichnung konfliktreich, so daß die ersten Geburtstage in beiden Ländern nicht zum Anlaß für Feierlichkeiten genommen wurden. Die Gründe sind naheliegend, und die verschiedenen Beiträge in diesem Sammelband beleuchten sie aus unterschiedlichen Perspektiven: In vielerlei Hinsicht war der Vertrag ein improvisiertes Gebilde, der Ausdruck eines doppelten Mißerfolges und der Prolog zu einer paradoxen Situation. Er bildete zum einen eine Art von Kompensation für den durchgefallenen "Plan Fouchet«. De Gaulle versuchte zwischen Frankreich und Deutschland Beziehungen in bilateraler Form auf die Beine zu stellen, die ihm im multilateralen Rahmen der Europäischen Gemeinschaft (EG) versagt worden waren. Da der Vertrag folglich nur eine Notlösung war, war es offensichtlich nicht ganz einfach, aus ihm ein Objekt der Erinnerung zu machen. Bleibt aber die Frage, ob ein Ersatzmittel würdig genug sein kann, um feierlich begangen zu werden?

Zum anderen erschwerte die transatlantische Ausrichtung der Präambel, die der Bundestag wenige Monate später hinzufügte, die Ausgangslage. Der enttäuschte General bemerkte in seinem legendär gewordenen Urteil, daß die Verträge wie Rosen und junge Mädchen seien und nur kurze Zeit Bestand hätten. Der Rückzug von Konrad Adenauer und die Emennung des "Atlantikers « Ludwig Erhard zu seinem Nachfolger sind Ereignisse, die den zweiten Mißerfolg bekräftigen, jenen vom Geist des 22. Januars. Aus allen diesen Vorkommnissen resultierte ein großes Paradox: Der Vertrag von 1963 leitete eine Periode ein, die von sehr schlechten Beziehungen zwischen Frankreich und der Bundesrepublik geprägt war. Die äußeren Umstände begünstigten folglich wenig den Hang zu Feierlichkeiten, die einzig einen deutschfranzösischen Mißerfolg hätten konstatieren können, der selbst aus dem Scheitern europäischer Einigungspläne hervorgegangen war.

Zum ersten Geburtstag im Januar 1964 erwähnte die französische Presse den Vertrag nur im Zusammenhang mit dem Befremden des bundesdeutschen Außenministers, der sich über die offizielle Anerkennung der Volksrepublik China durch Frankreich erstaunt zeigte, weil diese Entscheidung mit der Bundesrepublik nicht abgesprochen gewesen sei, so wie es der ein Jahr zuvor unterzeichnete Vertrag für solche Fälle eigentlich vorsah. Das Zusammentreffen von de Gaulle und Erhard im Jahre 1965 gab Anlaß zu der Hoffnung, daß sich das Klima zwischen beiden Ländern wieder verbessern könnte. Doch bereits 1966 trübte sich diese Zuversicht wieder ein, denn der Geburtstag fiel mit der Konferenz von Luxemburg zusammen, auf der eine Lösung für die "Krise des 
leeren Stuhls« gesucht werden sollte, die von Frankreich ausgelöst worden war. Die verabschiedete Entschließung erlaubte zwar einen Ausweg aus der verfahrenen Situation, doch konnte sie nicht über die Enttäuschung auf deutscher Seite über die französische Haltung hinwegtäuschen. Im Januar 1968 sprach die französische Presse mehr über den Vietnamkrieg und die TetOffensive als über den fünften Geburtstag des Vertrages. Nichtsdestotrotz erschienen einige aufschlußreiche Artikel, die sich mit dem Zustand der deutsch-französischen Beziehungen beschäftigten. P.-T. Franceschini bediente sich des blumigen Bildes von de Gaulle, um die Erfolge und Mißerfolge abzuwägen:

Der im Élysée-Vertrag genährte französische Traum von einer 'gemeinsamen Politikı lebte nur so kurz wie die Rosen. Die durch den Vertrag von 1963 eingeleitete institutionalisierte Kooperation bewies jedoch, daß sie dem Vertrag eine Langlebigkeit verleihen konnte. ${ }^{3}$

Zudem wird in dem Artikel auf die Erfolge hingewiesen, die sich aus der Gründung des Deutsch-Französischen Jugendwerkes für den Jugendaustausch zwischen beiden Ländern ergeben hatten. Weiterhin wird die sunumkehrbare« Dimension der Zusammenarbeit hervorgehoben, die sich aus den - nicht immer gut vorbereiteten - deutsch-französischen Gipfelgesprächen ergeben habe. Sie hätten für die beiden Regierungen einen Rahmen und eine Arbeitsatmosphäre geschaffen, auf der aufgebaut werden könne.

\section{Ein politischer Wille?}

Es bedurfte des Rücktritts von de Gaulle, immerhin einer der beiden Unterzeichner des Vertrages, um dem Vertrag neues Leben einzuhauchen. Die Beziehungen zwischen den beiden Staaten verbesserten sich in der Zeit Pompidou/Brandt, nicht zuletzt deshalb, weil ersterer den Beitritt Großbritanniens zur Europäischen Gemeinschaft akzeptierte, den die Bundesrepublik seit längerem gefordert, den de Gaulle jedoch immer abgelehnt hatte. Die deutschfranzösischen Gipfeltreffen wurden zu einem nützlichen Element der Europäischen Integration, da die eingeübten Arbeitssitzungen nun auch zu Ergebnissen führten. Nachdem auf der Haager Konferenz im Dezember 1969 beschlossen worden war, einen Stufenplan für die Errichtung einer Wirtschafts- und Währungsunion auszuarbeiten und dieser in Form des sogenannten »WernerPlans « im Oktober 1970 vorgelegt worden war, einigten sich der französische Staatspräsident und der Bundeskanzler zum achten Geburtstag des Élysée-

3 P.-T. FRANCESCHINI, Cinq ans de traité franco-allemand. Une coopération irréversible, in: Le Monde, 21./22. Januar 1968. 
Vertrages auf einen Kompromiß, der diesen nach dem luxemburgischen Ministerpräsidenten Pierre Werner genannten Plan zwar abschwächte, ihm jedoch die Möglichkeit zur Weiterentwicklung einräumte. Im Bezug auf den eventuellen Beitritt Großbritanniens und die Zukunft Europas wies Georges Pompidou auf die vorteilhafte Wirkung der Zeit hin und brachte seinen Wunsch zum Ausdruck, daß keine Sentimentalitäten mehr die Beziehungen zwischen den Mitgliedern der Europäischen Gemeinschaft und auch zwischen Frankreich und der Bundesrepublik dominieren würden. Um diese Vorstellung besser zu illustrieren, zitierte er den französischen Dichter Arthur Rimbaud:

Ah! que le temps vienne Où les cœurs s'éprennent.
Ah! Möge die Zeit kommen In der sich die Herzen verlieben.

Es handelte sich hier zweifellos um ein erstes Zeichen des Versuchs, einen Erinnerungsort aufzubauen, einen Raum, dem die Verbindung zwischen Vergangenheit, Gegenwart und Zukunft gelingt. Der neunte Geburtstag im Januar 1972 wurde zum Anlaß für die Unterzeichnung des Vertrages über die Erweiterung Europas genommen, mit dem der Beitritt Großbritanniens zur EG besiegelt wurde. Zum zehnten Geburtstag 1973 verliehen Pompidou und Brandt ihren feierlichen Ansprachen besondere Wärme. Der französische Präsident erinnerte an die wesentliche Funktion einer gelungenen Gedenkfeier, die der Zukunft zugewandt sein müsse, ob es sich nun um die Europäische Integration im Westen oder die Öffnung gegenüber dem Osten und der Entspannungspolitik handele:

Es bleibt zu hoffen, daß dieser Geburtstag nicht nur feierlich an die Vergangenheit erinnem will, sondern zu einem neuen Markstein unserer Zusammenarbeit, unseres Beitrages zur Europäischen Union, unserer Bemühungen um einen dauerhaften Frieden auf unserem gesamten Kontinent wird.

Nun begann die Zeit, in der der Vertrag zunehmend mystifiziert wurde, wie die Überschrift eines Artikels von Maurice Delarue in Le Monde vom 21./22. Januar 1973 belegt: "Die Versöhnung von Galliern und Germanen«. Zum gleichen Zeitpunkt entwickelte sich der erste Mythos: Alles habe mit de Gaulle und Adenauer begonnen. Mit dieser Interpretation wurde jedoch die Wende von 1950 vergessen, die mit der Annäherung zwischen Adenauer und Schuman verbunden ist.

Der zweite Mythos besteht darin, daß in den Beziehungen zwischen den beiden Ländern seit 1963 alles zum Besten steht, was die Ära de Gaulle-Erhard vergessen macht. Es ist wohl bekannt, da $\beta$ das Vergessen integraler Teil der Erinnerung ist, daß Mythen notwendig sind, um eine solide kollektive Er-

4 Willkommensansprache von Georges Pompidou aus Anlaß des Besuches von Willy Brandt im Élysée-Palast am 22. Januar 1973. 
innerung zu schaffen und daß der Prozeß der Mystifikation durch eine gezielte Auswahl der Erinnerungen begleitet wird. Es gilt jene auszulesen, die den meisten Sinn geben und die am stärksten zur Konstruktion von Symbolen beitragen.

Aus französischer Sicht ist Charles de Gaulle als Symbol der deutschfranzösischen Versöhnung naturgemäß stärker als Robert Schuman, weil er der "Held" war, der sich dem nationalsozialistischen Deutschland widersetzt hatte. Seine Vergangenheit als Chef der Résistance gab der Versöhnung nur noch weitere symbolische Kraft, doch bedurfte es der Zeit in diesem symbolbildenden Prozeß. Und diese Zeit war nach dem Rücktritt und dem Tod des Generals um so mehr gekommen, als sich die "leere Hülle« des Vertrages nach der konfliktreichen Periode zwischen 1963 und 1969 in der Ära Pompidou/Brandt langsam zu füllen begann. Wir können hier nun eine interessante Zeitenfolge beobachten: Zum einen trat der Zeitfaktor wieder auf den Plan, denn es bedurfte einer angemessenen Zeitdauer, damit sich die Erinnerung verwurzeln konnte. Dieser Prozeß wurde durch das Ableben de Gaulles und den Beginn seiner »Heroisierung" erleichtert. Zum anderen mußte die Zukunft der deutsch-französischen Beziehungen symbolisch unterfüttert werden, damit die Geschichte des Vertrages einen Platz in der Erinnerung findet. Aus diesem Grund konnte der Vertrag erst nach dem Tod des Helden Perspektiven einer gemeinsamen Zukunft schaffen.

In der Ära Giscard/Schmidt entwickelte sich die deutsch-französische $\mathrm{Zu}$ sammenarbeit auf beachtliche Weise und spielte eine entscheidende Rolle bei den Fortschritten der Europäischen Integration. Es ließe sich folglich vermuten, daß dieser Periode bei der Verwandlung des Vertrages von 1963 in einen Erinnerungsort eine bedeutende Funktion zukam. Wenn auch der Vertrag zu einem wirklichen deutsch-französischen Entscheidungs- und Initiativrahmen für das Zusammenwachsen Europas (Europäischer Rat, Europäisches Währungssystem, Direktwahl zum Europaparlament) avancierte, so wurden seine Geburtstage jedoch nicht eigens mit Glanz und Gloria begangen. Viel mehr waren die deutsch-französischen Gipfeltreffen von einer arbeitsamen Atmosphäre geprägt, die wenig Platz für feierlichen Pomp ließ. Valéry Giscard d'Estaing, der von sich das Bild eines Modernisierers zu pflegen beliebte, war eher der Zukunft als der Vergangenheit zugewandt und wollte sogar dem Nationalfeiertag am 8. Mai, dem Tag der Kapitulation des "Dritten Reiches", ein Ende setzen. Mit seiner Abschaffung gedachte er eine Geste zugunsten der Freundschaft zwischen den beiden Ländern zu tun. Diese Idee verbesserte die Beziehungen aber in keiner Weise, da sie bereits exzellent waren, stattdessen löste er mit ihr einen Streit mit den Widerstandskämpfern und den Kriegsteilnehmern aus. Valéry Giscard d'Estaing war kein Mann historischer Symbole und sein Wille zur Vervielfältigung der Kontakte mit dem Nachbarn östlich des Rheins ging nicht mit dem Ziel einher, deutsch-französische Erinnerungs- 
orte zu schaffen. Zum 15. Geburtstag im Jahre 1978 waren keine besondere Vorkehrungen getroffen worden, so daß der Historiker Gilbert Ziebura schrieb:

Wir haben es mit einem Paradox zu tun: wir leben mit dem Vertrag, ohne uns an seine Existenz zu erinnem [...]. Er ist erstart und zur Routine geworden, ein Monument, das Patina ansetzt.

Die Dynamik der deutsch-französischen Zusammenarbeit war abgetrennt von jeglichem Symbolisierungsprozeß, hingegen hatte die Zeit Wirkung gezeigt, so $\mathrm{da} B$ die regelmäßigen Treffen zwischen den beiden Regierungen ihre Früchte trugen. Das Monument war weiterhin da, jedoch erstartt und seines Sinnes enthoben. Ein Erinnerungsort verlangt jedoch danach, daß eine Verbindung zwischen der Patina der Vergangenheit und den Hoffnungen auf die Zukunft hergestellt wird.

\section{Die Suche nach den Symbolen}

Während der ersten Jahre seines Septennats trachtete François Mitterrand nicht danach, das Monument mit einer spezifischen Symbolik zu schmücken. Eine Wende vollzog sich erst aus Anlaß des 20. Geburtstages im Januar 1983 mit seiner Rede vor dem Bundestag am 20. Januar. Mitterand befürwortete die Stationierung der amerikanischen Pershing- und Cruise Missiles-Raketen in Europa für den Fall, daß die Verhandlungen mit der UdSSR über den Abzug der SS 20-Raketen zu keinem Ergebnis führten. ${ }^{6}$ Mit dieser Haltung unterstiitzte er die Sicherheitspolitik von Helmut Kohl und bezog im Streit innerhalb der Bonner Sozialdemokratie Position für Helmut Schmidt, der den NATO-Doppelbeschluß gegen die Mehrheit in seiner eigenen Partei durchgesetzt hatte und wenige Monate später als Kanzler zurücktreten mußte. Um die französische Solidarität für die bundesdeutsche Verteidigungspolitik feierlich zu proklamieren, wählte Mitterrand ein ganzes Ensemble von konvergierenden Symbolen: ein Datum aus der Vergangenheit, einen runden Geburtstag des Vertrages, der für die deutsch-französische Versöhnung steht, einen Ort, den Deutschen Bundestag, das Herz der bundesdeutschen Demokratie, eine Funktion - der Präsident der Französischen Republik sprach in diesem herausgehobenen Ort der Bundesrepublik. Gleichzeitig nutzte er die Gelegenheit, um je-

5 Gilbert ZiebURA, La naissance du traité, in: Documents (numéro spécial), November 1978, S. 9-20, hier S. 10.

- Vgl. Hélène MIARD-DelaCroiX, Les relations franco-allemandes, in: Serge BERSTEIN, Pierte Milza, Jean-Louis BianCo (Hg.), François Mitterrand. Les années de changement (1981-1984), Paris 2001, S. 295-310, hier S. 305. 
nen Ehre zu erweisen, die sich für die deutsch-französische Annäherung und die Europäische Integration eingesetzt hatten: Auf deutscher Seite erinnerte er selbstverständlich an Konrad Adenauer, aber auf französischer Seite rief er nicht alleine die Rolle von Charles de Gaulle ins Gedächtnis zurück, sondern auch jene von Jean Monnet und Robert Schuman. Auf diese Weise holte er den Gründungsakt von 1950 aus der Vergessenheit hervor und machte mit der Neigung ein Ende, alles 1963 beginnen zu lassen. Implizit gab er damit dem Werk von Konrad Adenauer eine größere historische Tiefe, war dieser auf bundesdeutscher Seite doch sowohl beim Schuman-Plan als auch beim ÉlyséeVertrag der Hauptdarsteller. ${ }^{7}$

Der Widerhall dieser Rede war beachtlich, Gefühle waren geweckt, die zwanzig Monate später noch stärker aufwallten: Am 22. September 1984 standen Kohl und Mitterrand in Verdun, vor dem Knochenhaus von Douaumont und hielten sich die Hand, um auf diese Weise die toten Soldaten zu ehren, die auf deutscher und französischer Seite während des Ersten Weltkrieges gefallen waren. Die gewählte Symbolik war nun ein andere. Der Einsatz einer unliebsamen Erinnerung verfolgte das Ziel, die Bildung einer engen Schicksalsgemeinschaft zu fördern, und kam damit einer doppelten Revolution in der Auswahl der Symbolik gleich. Von nun an verwies die französische Erinnerung an Verdun zum einen nicht mehr nur auf den Mut der Frontsoldaten ("poilus«), ihre Vaterlandsliebe und ihren Sieg, sondern wurde zu einer geteilten Erinnerung, einer binationalen Erinnerung, die sich auf die wechselseitigen Metzeleien bezog und als Lektion für den Frieden in Gegenwart und Zukunft verstanden werden sollte: Nie wieder, ein solches Gemetzel ist heute dank der deutsch-französischen Freundschaft nicht mehr vorstellbar. Zum anderen wurde eine negative Erinnerung an die Beziehungen zwischen Deutschland und Frankreich (das Blut von Verdun) in ein gemeinsames und positives Erinnerungserbe verwandelt, das der wechselseitigen Solidarität bei der Europäischen Integration dienen sollte. Bereits im Juli 1962 hatte es dafür einen Präzedenzfall gegeben, als erstmals de Gaulle und Adenauer den deutschfranzösischen Beziehungen über eine gemeinsame negative Erinnerung die bösen Geister der Vergangenheit auszutreiben gedachten, indem sie gemeinsam an einer religiösen Zeremonie in der Kathedrale von Reims teilnahmen, die das Symbol der im Ersten Weltkrieg zerstörten Bauwerke war. Diese Episode fand jedoch vor der Unterzeichnung des Vertrages statt, so daß sich die in Verdun gewählte Symbolik als wesentlich stärker erwies als jene in Reims.

Zehn Jahre später, im Jahre 1984, wandte sich die Erinnerung dem Zweiten Weltkrieg $\mathrm{zu}$, was für die beiden Länder wesentlich schwieriger zu bewerkstelligen war, da die Erinnerungen an Hitler und an Vichy die nationalen Ge-

Rede von François Mitterrand vor dem Bundestag am 20. Januar 1983; vgl. auch die Rede von Mitterrand im Élysée-Palast am 21. Januar 1983, in der von seinem Besuch im Museum in Rhöndorf sprach, das Adenauer gewidmet ist. 
wissen teilen. 50 Jahre nach der Landung der Alliierten in der Normandie, am 6. Juni 1994, stellte sich die Frage, ob es nicht endlich an der Zeit sei, zu den Feierlichkeiten auch hohe Vertreter der Bundesrepublik einzuladen. Die Antwort war schließlich negativ, als Ausgleich entschied jedoch Mitterrand, die deutschen Soldaten der deutsch-französischen Brigade und des 1992 gebildeten Eurocorps einzuladen, um sie am 14. Juli des gleichen Jahres über die Champs-Élysées defilieren zu lassen. Diese Entscheidung löste eine heftige Debatte in den Kreisen der ehemaligen Frontkämpfer und in der Presse aus. Mit Humor erinnerte Luc Rosenzweig in Le Monde vom 14. Juli daran, was an dieser Entscheidung des französischen Präsidenten neu war: Seit 1944 sei das einzige vielbeachtete Defilée eines Deutschen auf französischem Boden jenes von Karl Lagerfeld mit Claudia Schiffer gewesen. Als Zugeständnis an die sensiblen Gemüter wurde dann beschlossen, die deutschen Soldaten nicht zu Fuß paradieren zu lassen, um nicht schmerzhafte Bilder aus der Vergangenheit wieder aufzuleben zu lassen. Schließlich lenkten sie ihre Panzer über den Pariser Prachtboulevard und zeigten dabei ihre Köpfe aus den geöffneten Luken der Fahrzeuge. Die Erleichterung war groß, als die deutschen Soldaten mit herzlichem Applaus begrüßt wurden. Die Wette war damit gewonnen. Wieder einmal war eine für die Franzosen traumatische Erinnerung (die Aufmärsche von Wehrmachtsoldaten in den Straßen von Paris während der Besatzungszeit) in etwas Positives umgewandelt worden. Die Zeit half Wunden heilen, und dank eines sichtbaren politischen Willens war das Mittel gefunden, negative Erinnerungen in eine positive Erinnerung für die Zukunft umzuformen. Diese paradoxen Erinnerungsorte - Verdun im September 1984, die deutschen Soldaten auf den Champs-Élysées im Juli 1994 - standen nicht im Zusammenhang mit dem Vertrag von 1963, der erst im Jahr 2003 aus Anlaß seines 40. Geburtstages zur Geisteraustreibung genutzt wurde, die zuvor schon an anderen Orten so gut funktioniert hatte.

Nun wurde in Versailles eine Zeremonie organisiert, welche die Parlamente beider Länder vereinigte. Ein weiteres Mal evozierte der gewählte Ort negative Erinnerungen an eine gemeinsame Geschichte: das französische Trauma vom Januar 1871, als das Deutsche Kaiserreich im Spiegelsaal des Schlosses proklamiert worden war; das deutsche Trauma vom 28. Juni 1919, als die Unterschriften unter das "Diktat" von Versailles gesetzt worden waren. Mit den Feierlichkeiten im Jahr 2003 wollten die Politiker ihren beiden Völkern nun demonstriert, daß sie sich den Herausforderungen der Vergangenheit zu stellen und eine Erinnerungsgemeinschaft zu bilden haben, wenn sie ihre Schicksalsgemeinschaft bestätigen wollen.

Zudem wurden aus AnlaB des 40 . Geburtstages wichtige politische Entscheidungen getroffen. Nach einer Phase problembehafteter Beziehungen zwischen beiden Ländern und den dunklen Erinnerungen an den Gipfel von Nizza boten die Feierlichkeiten von 2003 die Gelegenheit, dem wcouple franco- 
allemand« einen neuen Schwung zu geben. Zum gleichen Zeitpunkt half die Irakpolitik des amerikanischen Präsidenten George W. Bush, wieder dichter zusammenzurücken. Im Januar 2003 fand diese neue Nähe ihren Ausdruck u.a. in den Erklärungen der beiden Länder gegen die Initiativen des Weißen Hauses. Die deutsch-französische Erklärung erinnerte nicht zufällig an die Notwendigkeit, die UNO-Resolutionen zu respektieren, und das internationale Recht besiegelte die Perspektivenübereinkunft der beiden Länder in der AuBenpolitik. Etwas voreilig glaubten Frankreich und Deutschland, da $B$ ihre parallelen Sichtweisen zur Grundlage für eine gemeinsame europäische Politik werden könnten, doch schnell zeigte sich Europa tief gespalten: Großbritannien, Spanien, Italien, Polen und vier weitere Länder verweigerten Deutschen und Franzosen ihre Solidarität und traten in der Irak-Frage an die Seite der USA. Schnell offenbarten sich die neuen Widersprüchlichkeiten, mit denen die Europäische Integration in Zukunft konfrontiert sein wird. Wenn es dem "couple franco-allemand" schlecht geht, geht es Europa schlecht, und das war schon immer so. Aber wenn es sich solide und einig zeigt, löst es Angst aus, was vorher so nicht der Fall war. Die Zeiten haben sich geändert, und viele (zukünftige) Mitglieder der Europäischen Union fürchten weniger das leadership der etwas weiter entfernten USA als die direkte und dichtere Gefahr einer deutsch-französischen Hegemonie.

Kann diese europäische Krise, die durch die Feierlichkeiten aus Anlaß des 40. Geburtstages ausgelöst wurde, den in Versailles begonnenen Prozeß gefährden, der darauf abzielt, aus dem Vertrag von 1963 einen paradoxen Erinnerungsort zu machen? Die Frage bleibt offen. Auch wenn George W. Bush den Irak-Krieg gewonnen hat, so steht es noch in den Sternen, ob er auch den Frieden erreichen wird. Die noch nicht abzusehenden Konsequenzen erlauben es seither Frankreich und Deutschland, auch bei ihren skeptischen Partnern Verständnis für eine Irak-Politik zu erlangen, die auf dem Respekt der UNO beruht, eine Politik, die übrigens von einer Mehrheit der europäischen Öffentlichkeit unterstützt wird. Wenn es Deutschen und Franzosen also gelingt, sich in ihren gemeinsamen Erklärungen weniger arrogant zu geben, so werden sie auch weiterhin eine beachtliche Kraft entwickeln, um die Europäische Integration voranzubringen. Auf jeden Fall bleibt die deutsch-französische Verbindung ungeachtet aller Erfolge und Mißerfolge in den Beziehungen zwischen den beiden Ländern stärker als irgendein Verhältnis zwischen anderen europäischen Ländern; dies gilt insbesondere auch für die Verbindungen zwischen Großbritannien, Spanien und Polen. In diesem Verhältnis zwischen Frankreich und Deutschland hat die Erinnerung mittlerweile dank geduldiger Bemühungen einen Platz eingenommen, der eine emotionale Verbindung zwischen Vergangenheit, Gegenwart und Zukunft garantiert.

Es scheint heute unmöglich, eine definitive Antwort auf die eingangs gestellte Frage zu geben, ist die Geschichte der deutsch-französischen Erinne- 
rung doch im Fluß und noch nicht abgeschlossen. Einige abschließende Bemerkungen sollten trotzdem erlaubt sein. Zuerst ist es nicht die Rolle des Historikers, einen Erinnerungsort zu konstruieren bzw. Ratschläge zu geben, wie ein solcher zu konstruieren ist. Er sollte sich auf die Beobachtung beschränken, und es ist ihm ein leichtes $z u$ konstatieren, daß verschiedene Erinnerungsorte "getestet" wurden, um das deutsch-französische Paar zu festigen: Reims 1962, der Bundestag 1983, Verdun 1984, die Champs-Élysées 1994 und Versailles 2003. Auf welche Weise kann sich der Élysée-Vertrag von 1963 dieser Erinnerung bemächtigen? Das Jahr 2003 hat zum einen gezeigt, $\mathrm{da} B$ ihm alleine dazu die Kraft fehlt, denn der Vertrag als immaterieller Erinnerungsort annektierte mit Versailles einen materiellen Erinnerungsort und spielte mit den Symbolen, um eine Linie zwischen den Traumata der Vergangenheit, der deutsch-französischen Union der Gegenwart und der Zukunft Europas zu ziehen. Dagegen ist die regelmäßige Wiederkehr seines Geburtstages ein Vorteil des Vertrages im Vergleich zu den anderen deutsch-französischen Erinnerungsorten, denn der 22. Januar erinnert nicht nur an die Kooperation zwischen beiden Staaten, sondern auch - wie die verschiedenen Beiträge in diesem Band zeigen - an die Verbindungen zwischen beiden Gesellschaften. Wenn auch das Erlernen der Partnersprache ein ständiges Problem bleibt, so sind die Erfolge in den Bereichen Erziehung und Jugend nicht zu übersehen. Zudem haben sich die Verbindungen zwischen Wirtschaft, Wissenschaft und Hochschulwesen weiter verstärkt. Diese Veränderungen sind grundlegend.

Schließlich bleibt festzustellen, da $B$ sich ein Erinnerungsort nicht von oben verordnen läßt, sondern erarbeitet und erworben werden muß. Wie stark auch immer der politische Wille und das Wirken der Zeit sein mögen, um eine Erinnerungsgemeinschaft aufzubauen, um eine gemeinsame Geschichte in eine gemeinsame Erinnerung zu verwandeln, so braucht es trotz allem einer gesellschaftlichen Unterfuitterung und Aufnahmebereitschaft. Der Élysée-Vertrag hat in diesem Prozeß eine wichtige Rolle gespielt, denn er hat die wechselseitige Neigung auf beiden Seiten des Rheins gefördert, sich der anderen Gesellschaft zu öffnen.

Nachdem der Vertrag anfänglich durch einen doppelten politischen Mißerfolg gekennzeichnet gewesen war, aber zugleich einen historischen Kooperationsrahmen geschaffen hatte, kommt uns der Élysée-Vertrag heute wie ein Gebilde daher, das durchaus in der Lage ist, Symbole zu produzieren, die den Aufbau einer deutsch-französischen Gemeinschaft emotional unterlegen, ganz so wie ein richtiger Erinnerungsort. Aber für wie lange? Schwer zu sagen. Auf jeden Fall für eine längere Zeit, als es de Gaulle selbst vorgesehen hatte und als Rosen überdauern. 
